\title{
Xenooplasmic Transfer between Buffalo and Bovine Enables Development of Homoplasmic Offspring
}

\author{
Marcos Roberto Chiaratti, Christina Ramires Ferreira,, ${ }^{1,2}$ Flávio Vieira Meirelles, Simone Cristina Méo,,3 \\ Felipe Perecin, ${ }^{1,2}$ Lawrence Charles Smith, ${ }^{4}$ Márcio Leão Ferraz, ${ }^{5}$ Manoel Francisco de Sá Filho, \\ Lindsay Unno Gimenes, ${ }^{6}$ Pietro Sampaio Baruselli, ${ }^{6}$ Bianca Gasparrini, ${ }^{7}$ and Joaquim Mansano Garcia ${ }^{2}$
}

\begin{abstract}
Nuclear-mitochondrial incompatibilities may be responsible for the development failure reported in embryos and fetuses produced by interspecies somatic cell nuclear transfer (iSCNT). Herein we performed xenooplasmic transfer (XOT) by introducing 10 to $15 \%$ of buffalo ooplasm into bovine zygotes to assess its effect on the persistence of buffalo mitochondrial DNA (mtDNA). Blastocyst rates were not compromised by XOT in comparison to both in vitro fertilized embryos and embryos produced by transfer of bovine ooplasm into bovine zygotes. Moreover, offspring were born after transfer of XOT embryos to recipient cows. Buffalo mtDNA introduced in zygotes was still present at the blastocyst stage (8.3 vs. 9.3\%, $p=0.11)$, indicating unaltered heteroplasmy during early development. Nonetheless, no vestige of buffalo mtDNA was found in offspring, indicating a drift to homoplasmy during later stages of development. In conclusion, we show that the buffalo mtDNA introduced by XOT into a bovine zygote do not compromise embryo development. On the other hand, buffalo mtDNA was not inherited by offspring indicating a possible failure in the process of interspecies mtDNA replication.
\end{abstract}

\section{Introduction}

$\mathbf{O}$ OCYTE CYTOPLASM (OOPLASM) carries critical components for supporting the initial stages of development when the embryo itself shows limited transcriptional activity, including reprogramming factors with the capacity of remodeling a somatic nucleus into an undifferentiated one after somatic cell nuclear transfer (SCNT) (Wilmut et al., 1997). However, development failure is common after SCNT, especially after interspecies SCNT (iSCNT), and its causes remain widely unknown. SCNT generates reconstructed oocytes with a mixture of mitochondria from different origins (e.g., heteroplasmy), that often disappear to generate offspring that are in most cases homoplasmic for mitochondria donated by the host ooplast (Ferreira et al., 2007; Hiendleder et al. 2003, 2005; Meirelles et al., 2001; Steinborn et al., 1998).
Mitochondrial function is driven by the interaction of two genomes: the nuclear (nDNA) and the mitochondrial (mtDNA). Although the role of the mtDNA is to encode polypeptides that assemble the enzymatic subunits of oxidative phosphorilation (the pathway responsible for ATP in the mitochondrion), most of the polypeptides in oxidative phosphorilation as well as those regulating mtDNA replication and transcription are encoded by the nDNA. Thus, the polypeptides encoded by the nDNA must match specific sequences in the mtDNA (e.g., the displacement loop or d-loop) and the mtDNA-encoded polypeptides to enable mitochondrial function to occur normally; otherwise, the cell viability may be compromised (reviewed by Smith et al., 2005). In this regard, it is plausible to propose that the procedure of iSCNT leads to nuclear-mitochondrial incompatibilities that prevent embryo development and birth of

\footnotetext{
${ }^{1}$ Departamento de Ciências Básicas, FZEA-USP, Pirassununga-SP, Brazil.

${ }^{2}$ Departamento de Medicina Veterinária Preventiva e Reprodução Animal, FCAV-UNESP, Jaboticabal-SP, Brazil.

${ }^{3}$ Embrapa Pecuária Sudeste, São Carlos-SP, Brazil.

${ }^{4}$ CRRA, Université de Montréal, Saint-Hyacinthe-QC, Canada.

${ }^{5}$ Vida Reprodutiva Consultoria, Cravinhos-SP, Brazil.

${ }^{6}$ Departamento de Reprodução Animal, Universidade de São Paulo, São Paulo-SP, Brazil.

${ }^{7}$ DISCIZIA, Federico II University, Napoli, Italy.

The first two authors contributed equally to this article.
} 
healthy offspring (Chen et al., 2002; Gómez et al., 2009; Hua et al., 2007; Kitiyanant et al., 2001; Lanza et al., 2000; Lu et al., 2005; Mastromonaco et al., 2007; Saikhun et al., 2002). The issue of a nuclear-mitochondrial incompatibility has been studied using xenomitochondrial cybrid models produced by the fusion of a somatic cell with a cytoplast (e.g., a cell previously enucleated) of another species (Kenyon and Moraes, 1997; Trounce and Pinkert, 2007) and using SCNT to analyze effects during embryo/fetal development in both inter- (Chen et al., 2002; Gómez et al., 2009; Lanza et al., 2000; Loi et al., 2001; Mastromonaco et al., 2007) or intraspecific (Bowles et al., 2008; Ferreira et al., 2007; Hiendleder et al. 2003, 2005; Meirelles et al., 2001; Steinborn et al., 1998) animal models. However, various factors involved in SCNT success (e.g., ooplasmic factors involved in somatic nucleus dedifferentiation) could prevent proper conclusions. In this context, it would be preferable to study the relationship between nDNA and mtDNA by transfer of ooplasm between individuals during early stages of development. Herein we have transferred ooplasm donated by buffalo (Bubalus bubalis) ooplasts into bovine (Bos indicus) zygotes to study the mitochondrial inheritance in embryos and offspring.

\section{Materials and Methods}

The present study was approved by the Institutional Animal Care and Use Committee of the São Paulo State University (UNESP) at the Jaboticabal Campus (protocol number 017256-06). All chemicals and reagents used were purchased from Sigma-Aldrich Chemical Co. (St. Louis, MO, USA) unless otherwise stated. In vitro experimental procedures were performed in humidified incubators maintained at $38.5^{\circ} \mathrm{C}$ in air with $5 \% \mathrm{CO}_{2}$.

In vitro maturation (IVM), in vitro fertilization (IVF), ovum pickup (OPU), parthenogenetic activation, enucleation, electrofusion, in vitro culture (IVC) of embryos, and embryo transfer were performed as described in Ferreira et al. (2007). Briefly, oocytes obtained postmortem from the ovaries of crossbred cows (B. indicus nDNA and B. taurus mtDNA) were IVM for $24 \mathrm{~h}$ and after $12 \mathrm{~h}$ of IVF were denuded of cumulus cells by pipetting in $0.5 \%$ hyluronidase, and selected by the presence of the second polar body (2nd PB), which were used as recipient zygotes for XOT. Meanwhile, donor ooplasts obtained by OPU from 10 female buffaloes (B. bubalis) were subjected to IVM for $21 \mathrm{~h}$ in the presence of $50 \mu \mathrm{M}$ cysteamine and $0.3 \mathrm{mM}$ cistine, and selected by the presence of the first polar body (1st PB) after removal of cumulus cells. The metaphase II spindle and the 1st PB were removed by microsurgery, and enucleated oocytes parthenogenetically activated $26 \mathrm{~h}$ post-IVM. To provide space for donor ooplasts, recipient zygotes had the 2nd PB and surrounding cytoplasm removed by microsurgery. After 12 to $16 \mathrm{~h}$ postparthenogenetic activation, 10 to $15 \%$ of ooplasm from the donor ooplast was microinjected in the perivitelline space and electrofused to the recipient zygote. One ooplast was used as donor ooplasm to reconstruct up to seven recipient zygotes. Reconstructed embryos (referred to as "XOT group") were IVC, and development rates were assessed based on blastocyst development after 7 days of culture. This rate was compared to that of IVF embryos that were previously selected for 2nd PB before IVC (referred to as "IVF group") and to that of IVF embryos that received cytoplasm from bovine oocytes previously activated (referred to as "OT group"). The same procedure used to perform XOT was used to produce embryos in the OT group. Moreover, to evaluate development to term, three blastocysts obtained by XOT were nonsurgically transferred to the uterus of three previously synchronized recipient cows, and were naturally delivered after normal gestation length.

The sperm used for IVF and offspring were genotyped following recommendation of the International Society for Animal Genetics. Briefly, sperm and blood (from offspring) samples had total genomic DNA extracted following a standard procedure (Ferreira et al., 2007). The genetic identity between the calves and the sperm was determined by microsatellite analysis using 11 microsatellite markers (Genoa Biotecnologia AS, São Paulo, SP, Brazil).

The inheritance of the mtDNA introduced by XOT was assessed in zygotes (at the pronuclear stage), at day 7 of IVC (blastocyst stage) and in offspring (0, 10, 30, and 290 days after birth). Embryos were randomly sampled (nine zygotes and six blastocysts), washed in phosphate-buffered solution (PBS), and placed in $0.2-\mathrm{mL}$ polystyrene microtubes containing $1 \mu \mathrm{L}$ of $\mathrm{PBS}+0.1 \%$ polyvinyl-pyrrolidone (PVP), snap-frozen in liquid nitrogen, and stored at $-80^{\circ} \mathrm{C}$ until use. Embryos pertaining to the OT group (13 zygotes and 11 blastocysts) also had their total number of mtDNA copies analyzed to be used as control in the experiment. Tissue samples (blood, skin, muscle, and liver) were collected from offspring, placed in $1.8-\mathrm{mL}$ cryotubes, and stored at $-80^{\circ} \mathrm{C}$ until use. Moreover, eight oocytes were collected by OPU (see above) from each offspring (after 18 months of birth) and stored as described for embryos.

For molecular analysis, oocyte and embryo samples were treated as described by Chiaratti et al. (2010), whereas tissue samples had the genomic DNA extracted following a standard procedure (Ferreira et al., 2007). The proportion of buffalo mtDNA was determined by quantitative polymerase chain reaction (qPCR) using a discriminating (buffalo mtDNA) assay and normalization for a nondiscriminating (buffalo + bovine mtDNAs) assay (Ferreira et al., 2010). For discrimination of buffalo and bovine mtDNAs (GenBank: AY702618, AY126697 and AY526085), species-specific differences in their MT-RNR2 genes sequences were targeted. The nondiscriminating assay used the primers bMT3010-f (5'-GCCCTAGAACAGGGCTTAGT) and bMT3096-r (5'GGAGAGGATTTGAATCTCTGG) in combination with the TaqMan probe bMT3030-Fam (5'-FAM-AAGGTGGCAGAG CCCGGTAATTGC-BHQ1). The discriminating assay used the primers bbMT2971-f (5'-GCCTTAAAACAACTAATG AATTT, discriminating nucleotides are underlined) and bMT3096-r, and the probe bMT3030-Fam. qPCR reactions were performed in duplicate in a $20-\mu \mathrm{L}$ reaction containing $900 \mathrm{nM}$ of forward (discriminating or nondiscriminating assay) and reverse primers (Applied Biosystems, Foster City, CA, USA) $+250 \mathrm{nM}$ probe (Applied Biosystems $)+1 \times$ TaqMan Gene Expression Master Mix (Applied Biosystems) + $5 \mu \mathrm{L}$ of template. Cycling conditions consisted of a first step at $95^{\circ} \mathrm{C}$ for $15 \mathrm{~min}$ followed by 40 cycles of $95^{\circ} \mathrm{C}$ for $20 \mathrm{sec}$ and $63^{\circ} \mathrm{C}$ for $1 \mathrm{~min}$. The ABI PRISM SDS $7500 \mathrm{HT}$ Real-Time PCR System (Applied Biosystems) was used for qPCR reactions. Both discriminating and nondiscriminating assays were always run in the same PCR plate. The standard curve method was used to determine the percentage 
of buffalo mtDNA. Assay sensitivity was estimated to be $0.01 \%$ of buffalo mtDNA. The total number of copies mtDNA (buffalo + bovine) and the number of copies of buffalo mtDNA were also assessed in embryos (zygote and blastocyst stages) following the procedure described by Chiaratti et al. (2010).

Statistical analysis was performed using the SAS System (V8, Cary, NC, USA). Development rates were compared using $\chi^{2}$. The percentage of buffalo mtDNA and the number of mtDNA copies in embryos were compared between zygote and blastocyst stages using a Student's $t$-test. Differences with probabilities $(p)<0.05$ were considered significant. In the text, values are reported as mean \pm the standard error of the mean (SEM).

\section{Results and Discussion}

Interspecies SCNT is envisaged to rescue endangered animals whose oocytes are difficult to obtain. However, offspring generated by this procedure will contain mostly the mtDNA donated by the ooplast (Ferreira et al., 2007; Hiendleder et al. 2003, 2005; Meirelles et al., 2001; Steinborn et al., 1998). The use of ooplasts from a more abundant related species (e.g., slaughterhouse-derived cattle oocytes for endangered artiodactyls) could lead to incompatible interactions between the nDNA and the mtDNA (Bowles et al., 2008; Gómez et al., 2009; Kenyon and Moraes, 1997; Lanza et al., 2000; Loi et al., 2001; Trounce and Pinkert, 2007). In this regard, XOT is a valuable tool to study nuclearmitochondrial interaction between species.

The procedure of iSCNT has been used without much success. Most reports show that the embryo is capable of developing to the blastocyst stage or even until later stages but not to derive viable offspring (Chen et al., 2002; Gómez et al., 2009; Hua et al., 2007; Kitiyanant et al., 2001; Lanza et al., 2000; Lu et al., 2005; Mastromonaco et al., 2007; Saikhun et al., 2002). Two main factors related to the ooplasm could be taken into account to explain iSCNT inefficiency: (1) its incapacity for remodeling the somatic nucleus into a totipotent one, (2) an incompatibility between mtDNA and nDNA. Herein we focused on the second possibility, because an incompatibility between these two genomes could compromise the mitochondrion-dependent mechanism of ATP generation in the cell and therefore lead to a development arrest (Bowles et al., 2008; Gómez et al., 2009; Kenyon and Moraes, 1997; Lanza et al., 2000; Loi et al., 2001; Trounce and Pinkert, 2007). Our study was based on a model where 10 to $15 \%$ of buffalo ooplasts are transferred into bovine zygotes. Because we intended to evaluate interspecies mtDNA inheritance in offspring, XOT was chosen rather than iSCNT due to the low efficiency of the latter in generating live offspring (Chen et al., 2002; Gómez et al., 2009; Hua et al., 2007; Kitiyanant et al., 2001; Lanza et al., 2000; Lu et al., 2005; Mastromonaco et al., 2007; Saikhun et al., 2002). Moreover, introduction of amounts of ooplasm larger than 10-15\% might drastically modify the zygote's cytoplasm composition, leading to development arrest as well. On the other hand, introduction of somatic cytoplast might lead to a more stringent segregation of somatic mtDNA due to specific differences between somatic and ooplasmic mitochondria (Bowles et al., 2008; Ferreira et al., 2007).
In our study, embryo development was not compromised by the transfer of buffalo ooplasm into bovine zygotes. Blastocyst rate of IVF $(65 / 235,27.7 \%)$, OT $(75 / 314,23.9 \%)$, and XOT $(15 / 66,22.7 \%)$ embryos were not different $(p=0.53)$. Moreover, XOT did not prevent development to term after embryo transfer to recipient cows enabling birth of female twins (Fig. 1). Analysis of microsatellite markers confirmed that offspring were generated with the sperm used in IVF and twins were genetically identical, probably due to embryo splitting after transfer to recipient cows. Although previous studies have shown development to blastocyst after iSCNT using bovine ooplasts and buffalo nucleus (Kitiyanant et al., 2001; Lu et al., 2005; Saikhun et al., 2002), transfer of xenoembryos to surrogate females was not performed. Using a somatic cell model, studies on xenomitochondrial cybrids have shown that with increased evolutionary divergence a barrier is reached whereby the foreign mtDNA can no longer be maintained, so that cybrids are not viable (Kenyon and Moraes, 1997; Trounce and Pinkert, 2007). Moreover, there are significant evidences that increased evolutionary distance is detrimental to embryo/ fetal development after iSCNT (Bowles et al., 2008; Gómez et al., 2009; Lanza et al., 2000; Loi et al., 2001). Although the unaltered development to blastocyst and to term in our model indicates proper nuclear-mitochondrial interactions, another possible interpretation is that buffalo mtDNA does not interfere with development because (1) mitochondrial activity is low during the early stages of embryogenesis and (2) the majority ( 85 to $90 \%$ ) of the mitochondria in the XOT zygotes is of bovine origin.

Comparison between buffalo and bovine mtDNAs showed that they differ by $12 \%$ in sequence (based on Genbank sequences, see above), whereas the difference in the replication origin and in the d-loop reaches 7 and 19\%, respectively. This may compromise the subsistence of buffalo mtDNA once mitochondrial replication and transcription is initiated, and undermined by inefficient nuclear-mitochondrial interactions. To test this hypothesis we checked whether blastocysts produced by XOT retain the donor mtDNA (Fig. 2). The average number of buffalo mtDNA copies present at the zygote stage was $43,157 \pm 5708$ (range: 11,097 to 74,492 ) and

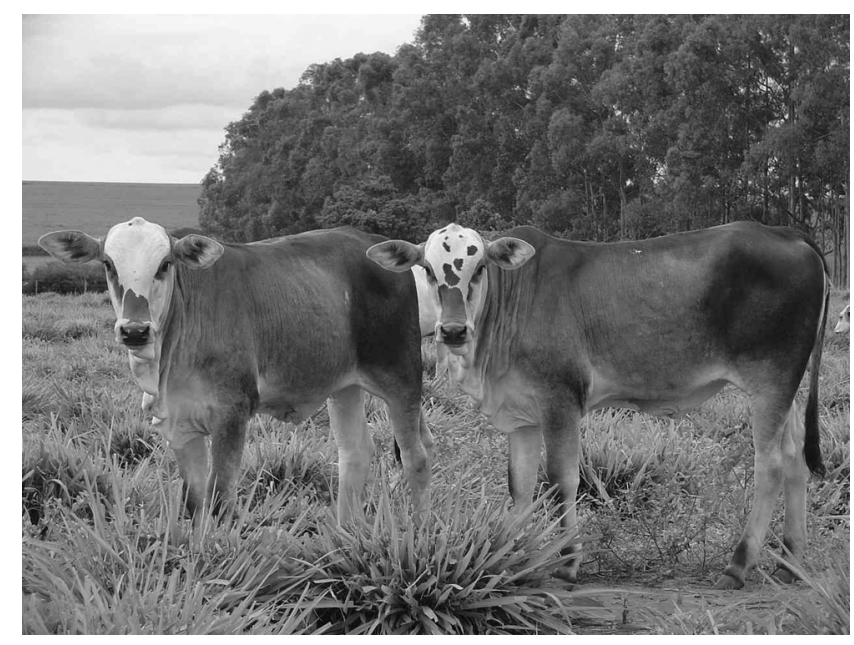

FIG. 1. Twin calves born after transfer of buffalo ooplasm into bovine zygotes. 

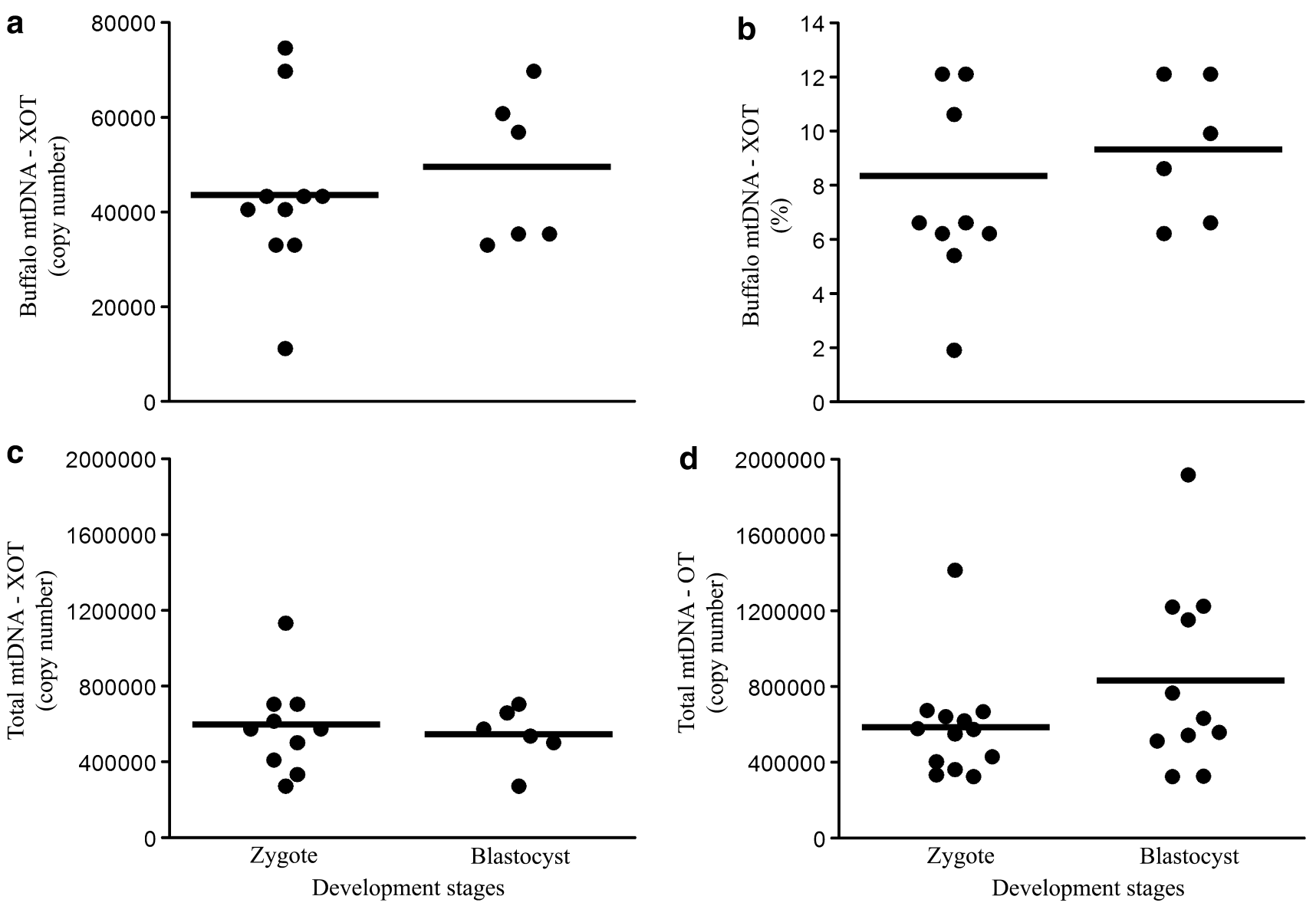

FIG. 2. Stable amounts of buffalo mtDNA during early development of XOT embryos. (a) Number of copies and (b) percentage of buffalo mtDNA, and (c) total (buffalo + bovine) number of copies of mtDNA in XOT embryos. (d) Total number of copies of mtDNA in OT embryos. Dots indicate the number of mtDNA copies in each embryo and bars represent the means. Comparisons between stages did not detect differences $(p>0.05)$.

the average number of total mtDNA was $580,636 \pm 76,545$ (range: 271,156 to $1,130,818$ ). Therefore, the percentage of buffalo mtDNA present in XOT zygotes was $8.3 \% \pm 1.26$ (range: $1.9-14.9 \%$ ), which is rather smaller than the amount of buffalo ooplast transferred (10-15\%). One explanation is that mitochondria distribution among donor and host zygotes was different, for example, more concentrated in the bovine zygote, leading to the introduction of less buffalo mtDNA than expected. In pigs, it is known that mitochondria are remodulated shortly after fertilization, leading to a heterogeneous cytoplasmic distribution during the one-cell stage (Sun et al., 2001).

In comparison to zygotes, buffalo blastocysts contained similar amounts of mtDNA molecules $(48,425 \pm 6,470$; range: $32,940-69,595 ; p=0.56$ ). Moreover, because no amplification of the total amount of mtDNA was observed between zygotes (see above) and blastocysts $(539,701 \pm 61,896$; range: $271,156-702,536 ; p=0.72)$, the percentage of buffalo mtDNA (blastocysts $=9.3 \% \pm 1.07$; range: $6.2-12.1 \%$ ) did not change $(p=0.11)$. A similar result was observed $(p=0.13)$ in control embryos subjected to OT using bovine ooplasm (OT group) (zygote $=580,636 \pm 77,963$ and blastocyst $=832,926 \pm$ 147,634; Fig. 2). Although mtDNA amounts do not change during in vivo preimplantation development (Kameyama et al., 2007; Pikó and Taylor, 1987; Smith et al., 2005; Stein- born et al., 1998), embryos cultured in vitro often show increased mtDNA amounts between zygote and blastocyst stages in a number of species (Chiaratti et al., 2010; Kameyama et al., 2007; May-Panloup et al., 2005; McConnell and Petrie, 2004; Mtango et al., 2008; Smith et al., 2005; Spikings et al., 2007) including after iSCNT within the Bos genus, for example, B. gaurus and B. taurus (Mastromonaco et al., 2007). Nonetheless, although variations in embryo in vitro culture systems may account for part of the differences in mtDNA levels (Kameyama et al., 2007; McConnell and Petrie, 2004; Mtango et al., 2008), complete lack of amplification of donor mtDNA in these XOT embryos during in vitro development may further reflect the nuclearmitochondrial incompatibilities (Bowles et al., 2008; Gómez et al., 2009; Kenyon and Moraes, 1997; Lanza et al., 2000; Loi et al., 2001; Trounce and Pikert, 2007) between the phylogenetically distant Bubalus and Bos genera (MacEachern et al., 2009). Moreover, detailed screening for buffalo mtDNA in offspring using a real-time protocol able to detect as little as $0.01 \%$ of buffalo mtDNA, was unsuccessful in identifying any vestige of buffalo mtDNA in somatic (blood, skin, muscle, and liver) and germline (oocytes) tissues of both calves born from XOT. Ferreira et al. (2010), using a similar approach to that described in the present work, have performed ooplasmic transfer between $B$. indicus and B. taurus cows and found 
that the introduced mtDNA was inherited by all offspring generated. Moreover, even when very small amounts of cytoplasm are transferred by ooplasmic transfer as performed in women in the past, the donor mtDNA can be detected in babies (Barrit et al., 2001; Brenner et al., 2000). Mitochondrial function is tightly dependent on polypeptides encoded by the nDNA that are imported by the mitochondrion after being translated in the cytoplasm. These polypeptides promote both replication and transcription of the mtDNA, and assemble the enzymatic subunits of the oxidative phosphorilation together with polypeptides encoded by the mtDNA (reviewed by Smith et al., 2005). Thus, the complete disappearance of the donor mitochondria in all postnatal tissues further indicates that buffalo mtDNA were unable to establish a functional interaction with the bovine nDNA, thereby preventing its propagation during development.

In conclusion, transfer of buffalo ooplasm into bovine zygotes enables birth of healthy offspring that do not inherit the mtDNA introduced. These results are relevant to a better understanding of nuclear-mitochondrial interactions between species. Further studies addressing this issue will be necessary to clarify the consequences of genetic diversity on iSCNT and its potential therapeutic applications, such as in deriving interspecific human embryonic stem cells.

\section{Acknowledgments}

The authors gratefully acknowledge the help provided by Dr. M.S. Miranda, J.R. Sangalli, and R. Vantini. This work was supported by research grants (2002/05054-7, 2004/01841-0 and 2006/59074-0) from FAPESP, São Paulo, SP, Brazil.

\section{Author Disclosure Statement}

The authors declare that no conflicting financial interests exist.

\section{References}

Barrit, J.A., Brenner, C.A., Malter, H.E., et al. (2001). Mitochondria in human offspring derived from ooplasmic transfer. Hum. Reprod. 16, 513-516.

Bowles, E.J., Tecirlioglu, R.T., French, A.J., et al. (2008). Mitochondrial DNA transmission and transcription after somatic cell fusion to one or more cytoplasts. Stem Cells 26, 775-782.

Brenner, C.A., Barrit, J.A., Willadsen, S., et al. (2000). Mitochondrial DNA heteroplasmy after human ooplasmic transplantation. Fertil. Steril. 74, 573-578.

Chen, D.Y., Wen, D.C., Zhang, Y.P., et al. (2002). Interspecies implantation and mitochondria fate of panda-rabbit cloned embryos. Biol. Reprod. 67, 637-642.

Chiaratti, M.R., Bressan, F.F., Ferreira, C.R., et al. (2010). Embryo mitochondrial DNA depletion is reversed during early embryogenesis in cattle. Biol. Reprod. 82, 76-85.

Ferreira, C.R., Meirelles, F.V., Yamazaki, W., et al. (2007). The kinetics of donor cell mtDNA in embryonic and somatic donor cell-derived bovine embryos. Cloning Stem Cells 9, 618-629.

Ferreira, C.R., Burgstaller, J.P., Perecin, F.P., et al. (2010). Pronounced segregation of donor mitochondria introduced by bovine ooplasmic transfer to the female germ-line. Biol. Reprod. 82, 563-571.

Gómez, M.C., Pope, C.E., Ricks, D.M., et al. (2009). Cloning endangered felids using heterospecific donor oocytes and interspecies embryo transfer. Reprod. Fertil. Dev. 21, 76-82.
Hiendleder, S., Zakhartchenko, V., Wenigerkind, H., et al. (2003). Heteroplasmy in bovine fetuses produced by intra- and inter-subspecific somatic cell nuclear transfer: neutral segregation of nuclear donor mitochondrial DNA in various tissues and evidence for recipient cow mitochondria in fetal blood. Biol. Reprod. 68, 159-166.

Hiendleder, S., Zakhartchenko, V., Wolf, E. (2005). Mitochondrial and the success of somatic cell nuclear transfer cloning: from nuclear-mitochondrial interactions to mitochondrial complementation and mitochondrial DNA recombination. Reprod. Fertil. Dev. 17, 69-83.

Hua, S., Zhang, Y., Song, K., et al. (2007). Development of bovine-ovine interspecies cloned embryos and mitochondria segregation in blastomeres during preimplantation. Anim. Reprod. Sci. 105, 245-257.

Kameyama, Y., Filion, F., Yoo, J.G., et al. (2007). Characterization of mitochondrial replication and transcription control during rat early development in vivo and in vitro. Reproduction 133, 423-432.

Kenyon, L., Moraes, C.T. (1997). Expanding the functional human mitochondrial DNA database by the establishment of primate xenomitochondrial cybrids. Proc. Natl. Acad. Sci. USA 94, 9131-9135.

Kitiyanant, Y., Saikhun, J., Chaisalee, B., et al. (2001). Somatic cell in buffalo (Bubalus bubalis): effects of interspecies cytoplasmic recipients and activation procedures. Cloning Stem Cells 3, 97-104.

Lanza, R.P., Cibelli, J.B., Diaz, F., et al. (2000). Cloning of an endangered species (Bos gaurus) using interspecies nuclear transfer. Cloning 2, 79-90.

Loi, P., Ptak, G., Barboni, B., et al. (2001). Genetic rescue of an endangered mammal by cross-species nuclear transfer using post-mortem somatic cells. Nat. Biotechnol. 19, 962-964.

Lu, F., Shi, Deshu, S., et al. (2005). Development of embryos reconstructed by interspecies nuclear transfer of adult fibroblasts between buffalo (Bubalus bubalis) and cattle (Bos indicus). Theriogenology 64, 1309-1319.

MacEachern, S., McEwan, J., Goddard, M. (2009). Phylogenetic reconstruction and the identification of ancient polymorphism in the Bovini tribe (Bovidae, Bovinae). BMC Genomics 10, 177.

Mastromonaco, G.F., Favetta, L.A., Smith, L.C., et al. (2007). The influence of nuclear content on developmental competence of gaur $\times$ cattle hybrid in vitro fertilized and somatic cell nuclear transfer embryos. Biol. Reprod. 76, 514-523.

May-Panloup, P., Vignon X., Chrétien, M-F., et al. (2005). Increase of mitochondrial DNA content and transcripts in early bovine embryogenesis associated with upregulation of mtTFA and NRF1 transcription factors. Reprod. Biol. Endocrinol. 3, 65.

McConnell, J.M., Petrie, L. (2004). Mitochondrial DNA turnover occurs during preimplantation development and can be modulated by environmental factors. Reprod. Biomed. Online 9, 418-424.

Meirelles, F.V., Bordignon, V., Watanabe, Y., et al. (2001). Complete replacement of the mitochondrial genotype in a Bos indicus calf reconstructed by nuclear transfer to a Bos taurus oocytes. Genetics 158, 351-356.

Mtango, N.R., Harvey, A.J., Latham, et al. (2008). Molecular control of mitochondrial function in developing rhesus monkey oocytes and preimplantation-stage embryos. Reprod. Fertil. Dev. 20, 846-859.

Pikó, L., Taylor, K.D. (1987). Amounts of mitochondrial DNA and abundance of some mitochondrial gene transcripts in early mouse embryos. Dev. Biol. 123, 364-374. 
Saikhun, J., Pavasuthipaisit, K., Jaruansuwan, M., et al. (2002). Xenonuclear transplantation of buffalo (Bubalus bubalis) fetal and adult somatic cell nuclei into bovine (Bos indicus) oocyte cytoplasm and their subsequent development. Theriogenology 57, 1829-1837.

Smith, L.C., Thundathil, J., Filion, F. (2005). Role of the mitochondrial genome in preimplantation development and assisted reproductive technologies. Reprod. Fertil. Dev. 17, 15-22.

Spikings, E.C., Alderson, J., St John, J.C. (2007). Regulated mitochondrial DNA replication during oocyte maturation is essential for successful porcine embryonic development. Biol. Reprod. 76, 327-335.

Steinborn, R., Zakhartchenko, V., Jelyazkov, J., et al. (1998). Composition of parental mitochondrial DNA in cloned bovine embryos. FEBS Lett. 426, 352-356.

Sun, Q.Y, Wu, G.M., Lai, L., et al. (2001). Translocation of active mitochondria during pig oocyte maturation, fertilization and early embryo development. Reproduction 122, 155-163.
Trounce, I.A., Pinkert, C.A. (2007). Cybrid models of mtDNA disease and transmission, from cells to mice. Curr. Top. Dev. Biol. 77, 157-183.

Wilmut, I., Schnieke, A.E., McWhir, J., et al. (1997). Viable offspring derived from fetal and adult mammalian cells. Nature $385,810-813$.

Address correspondence to: Marcos Roberto Chiaratti Departamento de Ciências Básicas FZEA-USP, Pirassununga-SP, Brazil

E-mail: chiaramr@fcm.unicamp.br Christina Ramires Ferreira Departamento de Ciências Básicas FZEA-USP, Pirassununga-SP, Brazil

E-mail: christina@iqm.unicamp.br 